\title{
The effect of hyperglycaemia on pancreatic islets transplanted into rats beneath the kidney capsule
}

\author{
D.W.R.Gray, D.Cranston, P. McShane, R.Sutton and P.J.Morris \\ Nuffield Department of Surgery, University of Oxford, John Radcliffe Hospital, Oxford, UK
}

\begin{abstract}
Summary. The effect of hyperglycaemia on islet transplantation in the rat has been examined in two ways, using syngeneic transplantation of 400 islets to the kidney capsule and subsequent measurement of kidney insulin content as a measurement of B-cell survival. Firstly, islets were transplanted into either diabetic or normal rats, then 6 months later the composite kidney/islet graft was transplanted into a normal rat. The insulin content was measured after a further 6 months and was found to be significantly reduced in islets exposed to hyperglycaemia in the primary recipient. These findings are interpreted as showing that long-term exposure of islets to hyperglycaemia results in B-cell loss. In the second experi-
\end{abstract}

ment 400 islets were transplanted into either a long-term (6 months) diabetic or a normal rat. Two weeks later the composite kidney/islet graft was transplanted into a normal rat. The insulin content was measured after a further 6 months and no significant difference was found, whether the primary recipient was diabetic or not. These results are interpreted as showing that islet graft implantation is not impaired in longterm diabetic rats.

Key words: Hyperglycaemia, islet transplantation, diabetes, rat.

to hyperglycaemia for prolonged periods prior to transplantation.

\section{Materials and methods}

\section{Animals and induction of diabetes}

Dark Agouti (DA) rats (bred in the Biomedical Services Unit, John Radcliffe Hospital) were used as both donors and recipients. One sibling of paired littermate DA rats was made diabetic by i.v. injection of streptozotocin $65 \mathrm{mg} / \mathrm{kg}$, diabetes being confirmed by a repeated serum glucose of $>22 \mathrm{mmol} / 12$ weeks later.

\section{Isolation of pancreatic islets}

Pancreatic islets were isolated from 3 donors by an intraductal collagenase technique [3], handpicked and then transplanted as packets of 400 islets to the renal capsular space as previously described [2].

\section{Glucose and insulin measurements}

Serum glucose was measured every 2 weeks after islet transplantation in primary hosts, and prior to the removal of the composite grafts from secondary recipients (see below). The insulin content of compo- 


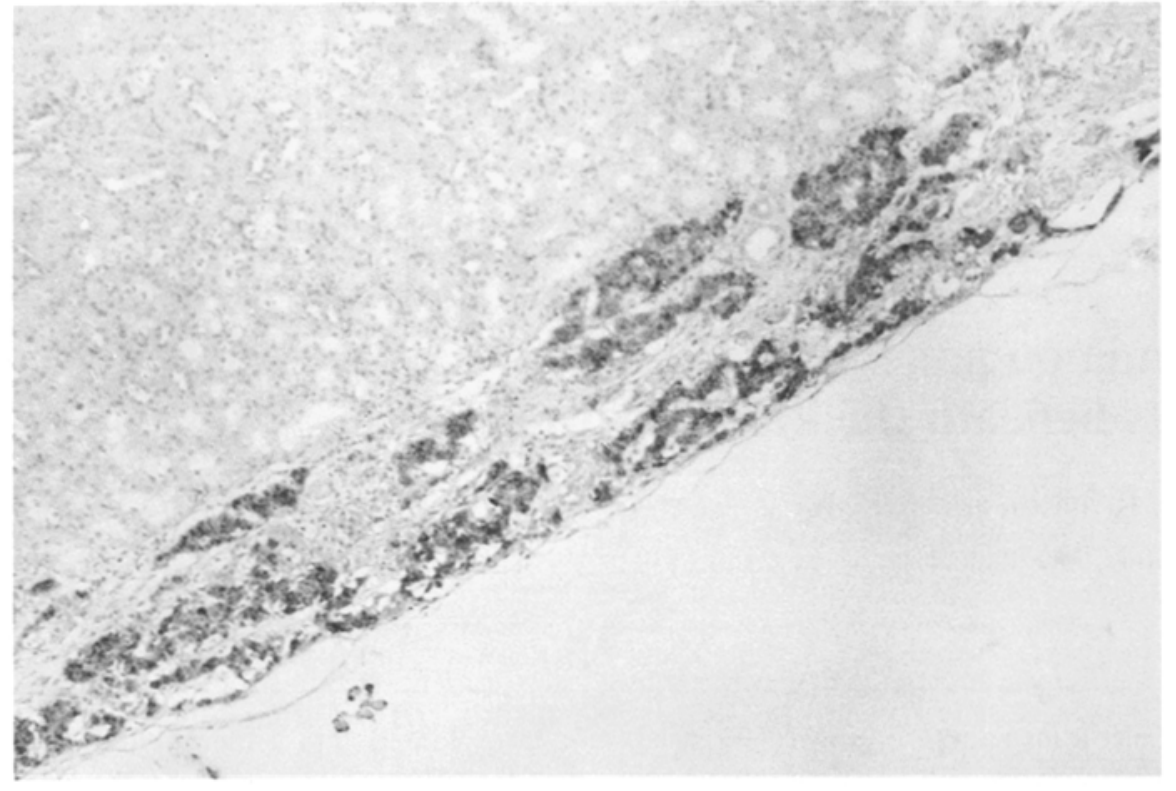

Fig. 1. Light micrograph of a kidney from a normoglycaemic rat given 400 islets transplanted under the kidney capsule. Six months later the combined kidney and islet graft was transplanted to a second normal syngeneic rat and left for a further 6 months before removal und processing for histological examination. Abundant dark-stained clumps of islet tissue are easily identified. Immunoperoxidase stain for insulin. Original magnification $\times 50$

site renal/islet grafts was measured by grinding the tissue in $\mathrm{CO}^{2}$ slush and acetone followed by extraction in acid alcohol and subsequent radioimmunoassay for insulin as previously described [2].

\section{Experimental protocols}

1. Effect of exposure of islets to long-term hyperglycaemia. Four hundred islets obtained from the same islet isolation were transplanted beneath the capsule of the left kidney of paired diabetic and normal littermate DA rats. The diabetic animals remained hyperglycaemic as the mass of islets transplanted was insufficient to reverse diabetes. Six months after transplantation the composite kidney/islet graft was removed from both the normal and diabetic animals and transplanted orthotopically to a normal DA rat (secondary recipient) as previously described [17]. Some composite grafts from normoglycaemic recipients were extracted for insulin content. After a further 6 months the composite kidney/islet grafts were removed from the secondary recipient and either processed for histological examination or insulin content.

2. Implantation of islets in long-term diabetic rats. One sibling of paired littermate DA rats was made diabetic and both animals were then maintained on standard laboratory diet. The serum glucose of both animals was measured every month for 6 months. After 6 months both the diabetic and normal paired littermate were given $400 \mathrm{DA}$ islets to the left renal subcapsule. The diabetic animals remained hyperglycaemic as the mass of islets transplanted was insufficient to reverse diabetes. Serum glucose was measured two weeks later and then the composite kidney/islet graft was removed from both the normal and diabetic animals and transplanted orthotopically to a normal DA rat (secondary recipient). After a further 6 months the composite kidney/islet grafts were removed from the secondary recipient and the insulin content measured. The right kidney was removed from the diabetic and normal primary recipients at 6 months and processed for histological examination. The stains used were immunoperoxidase stain for insulin and glucagon using a double antibody technique and also routine haematoxylin/eosin and periodic acid Schiff stains.

\section{Statistical analysis}

The results are expressed as the mean \pm SEM. Statistical analysis was performed using paired $t$ test.

\section{Results}

\section{Effect of exposure of islets to long-term hyperglycaemia}

Islet transplants were performed in 25 paired diabetic and normal rats. After islet transplantation one rat became normoglycaemic and, thus, this pair was excluded from further analysis. Due to death of one or both of the animals postoperatively, or from either infection or tumour formation 14 pairs were lost; usually, but not always, it was the diabetic rat that died. Four unpaired normoglycaemic rats were killed after 6 months and the insulin content of the composite kidney/islet graft was measured. The remaining unpaired animals that completed the experiment were used for histological analysis only. Thus, 10 pairs of composite kidney/islet grafts completed the experiment and 7 pairs were used for quantitative analysis of insulin content, the other 3 pairs being used for histological examination.

\section{a Islets grafted into normoglycaemic primary and second-} ary hosts. The normoglycaemic animals given 400 islets remained normoglycaemic and healthy. Examination of kidneys removed from normoglycaemic animals at 6 months revealed an easily identifiable patch of islet tissue. The insulin content of 4 composite grafts removed from normoglycaemic animals at this time was $97 \pm 26 \mathrm{mU}$.

Following transplantation of the composite islet/ kidney grafts to non-diabetic secondary hosts and examination after a further 6 months an easily identifiable patch of islet tissue was again seen, unchanged from the appearance after removal from the primary host 6 months earlier. The insulin content of the normoglycaemic cohort of the paired grafts $(n=7)$ was $71.8 \mathrm{mU} \pm 13.0$. Histological examination $(n=3)$ 


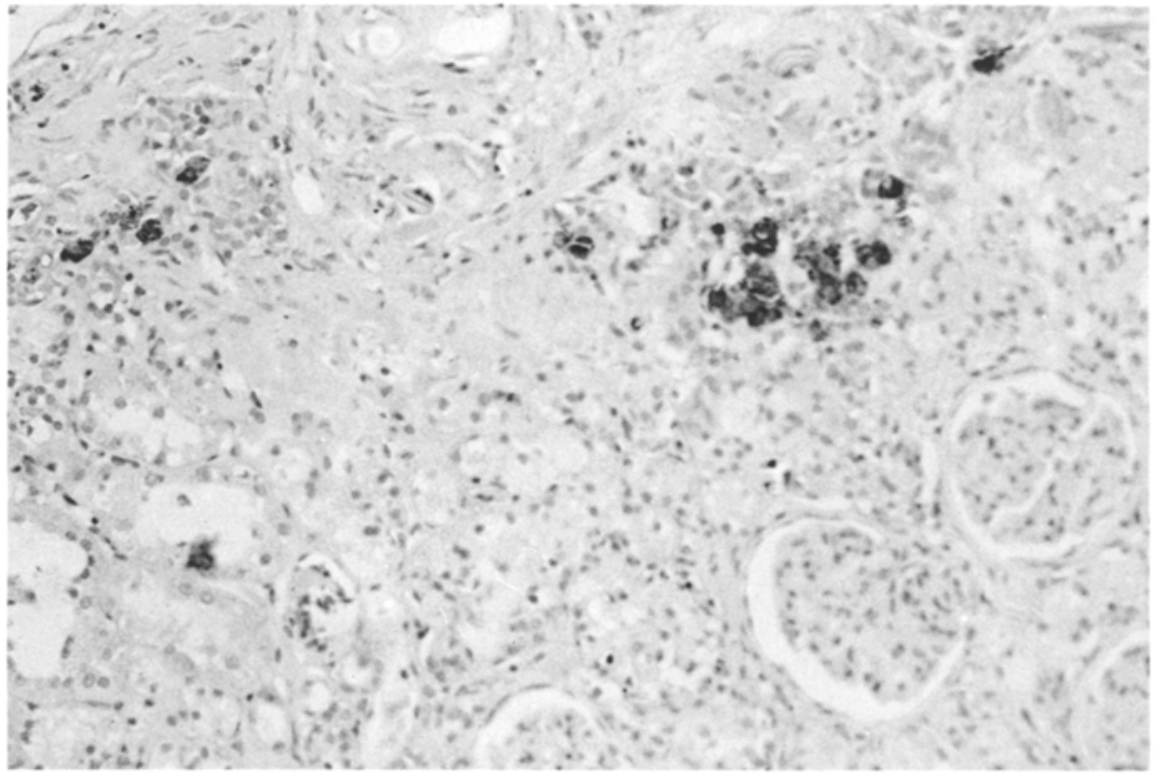

Fig. 2. Light micrograph of a kidney from a hyperglycaemic rat given 400 islets transpllanted under the kidney capsule. Six months later the combined kidney and islet graft was transplanted to a second notmal syngeneic rat and left for a further 6 months before removal and processing for histological examination. Occasional clusters of insulin-containing cells are identifiable interspersed between fibrous tissue.

Immunoperoxidase stain for insulin. Original magnification $\times 100$ showed abundant islet tissue as previously described [2] (Fig.1).

$b$ Islets grafted into hyperglycaemic primary hosts and normoglycaemic secondary hosts. Diabetic animals given $400 \mathrm{DA}$ islets remained hyperglycaemic $(>22 \mathrm{mmol} / \mathrm{l})$ throughout the six months and developed clinical features typical of chronic diabetes in the rat, such as wasting and abdominal distension. Kidneys removed after six months of hyperglycaemia showed an indistinct area of apparent scarring with no clearly defined islet tissue.

Following transplantation of the kidney/islet composite grafts into non-diabetic secondary hosts and examination after a further 6 months in the normoglycaemic environment the appearance of the islets varied between finding only a patch of scarring in some to a more "normal" but thin patch of islet tissue in others. The kidneys also tended to be smaller than normal. The insulin content of the hyperglycaemic cohort of the paired grafts $(n=7)$ was $11.9 \pm 3.1 \mathrm{mU}$, which was significantly different from the paired normoglycaemic group, which was $71.8 \pm 13(p<0.01)$. Histological examination $(n=3)$ showed scanty islet tissue with apparent fragmentation and some excess fibrous tissue, but islands of apparently normal islet tissue containing $B$ cells were visible (Fig.2). In some areas A cells were found, whereas B cells were absent (data not shown).

\section{Implantation of islets in long-term diabetic rats}

Twelve paired diabetic and normoglycaemic littermates were followed for 6 months. All the diabetic animals remained hyperglycaemic and there was a tendency for the hyperglycaemia to increase with time, so that at 6 months the plasma glucose was $>30 \mathrm{mmol} / 1$. These animals showed wasting and abdominal distension, as noted previously in long-term diabetic rats. Six of the diabetic animals died before the 6 months or during the operative procedure, thus, there were 6 paired rats that successfully underwent islet transplantation. The composite islet/kidney grafts were transplanted orthotopically into normal secondary recipients 2 weeks later, without losses. At the time of transplantation of the composite kidney/islet grafts the islets placed into the normoglycaemic animals were easy to identify, whilst the islet grafts placed in the hyperglycaemic recipients were much less distinct and harder to identify, having an appearance suggestive of scarring. However, when the composite kidney/islet grafts were removed from the secondary recipients of both groups 6 months later an easily identifiable patch of islet tissue was found in all cases. The insulin content of the composite grafts from normoglycaemic primary hosts was $112.1 \pm 31.3 \mathrm{mU}(n=6)$, compared to $95.8 \pm 19.9 \mathrm{mU}$ $(n=6)$ for islets transplanted to diabetic primary hosts $(p>0.5)$. Histological examination of kidneys removed from rats that had been hyperglycaemic for 6 months showed mesangial proliferation and nodular basement membrane thickening, in contrast to kidneys removed from non-diabetic littermates.

\section{Discussion}

Despite recent advances, clinical transplantation of isolated islets of Langerhans remains an elusive goal. Part of the problem lies in the mass of islets obtainable for transplantation. In syngeneic rodent models of islet transplantation multiple donors can be used, but this is not practical for transplantation of human islets or in models of islet transplantation in large animals. Transplantation of a less than optimal mass of islet tissue might be expected to result in reduced secretion of insulin and other hormones in response to glucose 
or other stimuli. There is abundant evidence that this is the case in models of islet autotransplantation in pancreatectomized large animals, despite the fact that fasting normoglycaemia can be reproduced [4-6]. Furthermore, recent studies in the dog [6] and monkey [4] have shown that many autografts will fail between 4 and 18 months after transplantation, and that the rapidity of failure is predicted by the insulin secretory capacity at 6 weeks [4]. This probably reflects the islet cell mass which has successfully implanted. This apparent failure of insulin secretion appears similar to the "exhaustion" seen clinically after subtotal pancreatectomy [7], and experimentally in partially pancreatectomized animals [8], although the exact cause remains unknown. One possible cause may be that a reduced mass of $B$ cells may be able to maintain fasting normoglycaemia, but at other times, when hyperglycaemia ensues, the chronic secretory overstimulation resulting from persistent hyperglycaemia results in eventual exhaustion.

Persistent chronic hyperglycaemia in the recipient may alter the microvascular circulation and thereby diminish the rate of revascularisation of the transplanted islets and hence the success of implantation. As the underlying abnormality of the complications of diabetes is thought to be a microangiopathy, transplantation of isolated islets of Langerhans or fetal pancreas has been proposed as a way of treating diabetes and preventing the onset of these complications. Free grafts of isolated pancreatic islets or fetal pancreas normally re-establish a blood supply within a few days [9]. Animal models of islet and fetal pancreas transplantation have met with remarkable success in terms of reversing experimentally induced and even naturally occurring diabetes [10]. However, in these animal models transplantation is usually performed after only a relatively short duration of diabetes, often less than a month.

The failure of recent clinical trials of both isolated islet transplantation $[11,12]$ and fetal transplantation [13], has raised the possibility that islet implantation and revascularization may be impaired by the microangiopathy invariably present in the long-term diabetic patients who are the recipients $[14,15]$. This is consistent with the common experience that if experimental animals are left diabetic for some months before islet transplantation is performed reversal of the diabetic state is much more difficult [14]. Again, it is possible that this difficulty is caused by impaired islet implantation secondary to microangiopathy, since it is known that microangiopathic changes are seen in organs such as the kidney [16] and eye [17] after 6 to 14 months of hyperglycaemia in animals such as the rat.

In order to examine the effect of prolonged hyperglycaemia on pancreatic islets transplanted beneath the kidney capsule we used a quantitative model of islet transplantation. To minimise variability in the experimental protocol we used matched littermate pairs of diabetic and normal rats, as the main source of variation in this model is variation in islet size from different islet isolations with consequent differences in insulin content [18]. We quantified the mass of insulin containing tissue present by measuring the insulin content. It is recognized that for this purpose the islets must be in a stable normoglycaemic environment for sufficient time to allow full granulation of the B cells, and for this reason the composite kidney/islet grafts were transplanted into normal syngeneic hosts and left for a full 6 months. The results of experiment 1 show that prolonged exposure to hyperglycaemia leads to actual loss of B cell mass. These findings are consistent with the results of autotransplantation of islets in large animal models, where animals with fasting normoglycaemia but non-fasting hyperglycaemia tend to fail after a few months [19]. More recently it has been shown that the prolonged production of hyperglycaemia by injection of glucose in partially pancreatectomised dogs resulted in the production of permanent diabetes [20]. The findings are in contrast to the lack of effect of short-term hyperglycaemia [2]. From the point of view of islet transplantation the results provide some encouragement, since they suggest that if sufficient islets can be implanted to produce euglycaemia then graft failure will not occur. Although it is recognised that transplanted free islets may behave differently to islets within the pancreas, these findings may have a greater relevence in diabetes, for they suggest a mechanism for the "exhaustion" seen after partial pancreatectomy and a possible contributory mechanism to the "honeymoon" effect seen in newly diagnosed diabetic patients.

Experiment 2 provides for the first time quantitative data on the survival of islets transplanted into long-term diabetic rats. The results showed that islet implantation into long-term (6 month) diabetic animals was not markedly impaired, compared to nondiabetic littermates, although the numbers are too small to be sure that a small difference was not present. It is arguable whether the microangiopathic changes seen in rat kidneys after 6 months of hyperglycaemia have any relevance for the clinical situation, where the changes develop over 15 or more years. Nevertheless, the histological features show some similarity with human diabetic nephropathy, as noted in the present experiment and previous studies. These changes have been invoked as an explanation for the increased difficulty in reversing diabetes that is experienced when recipient rats that have been hyperglycaemic for some months are given islet or fetal transplants $[14,15]$. The present study does not support the concept that impaired implantation due to microangiopathy is the explanation for these observations.

Acknowledgements. This work was supported by grants from the Medical Research Council of the U.K., the British Diabetic Association, and the Juvenile Diabetes Foundation International. 


\section{References}

1. Unger RH, Grundy S (1985) Hyperglycaemia as an inducer as well as a consequence of impaired islet cell function and insulin resistance: implications for the management of diabetes. Diabetologia 28: $119-121$

2. Gray DW, McShane P, Morris PJ (1986) The effect of hyperglycaemia on isolated rodent islets transplanted to the kidney capsule site. Transplantation 41: 699-703

3. Sutton R, Peters M, McShane P, Gray DWR, Morris PJ (1986) Isolation of rat pancreatic islets by ductal injection of collagenase. Transplantation 42: 689-691

4. Sutton R, Gray DW, McShane P, Peters M, Morris PJ (1987) Metabolic efficiency and long-term fate of intraportal islet grafts in the cynomolgus monkey. Transplant Proc 19:3575-3576

5. Alderson D, Walsh TN, Farndon JR (1984) Islet cell transplantation in diabetic dogs: studies of graft function and storage. $\mathrm{Br} \mathrm{J}$ Surg 71: 756-760

6. Alejandro R, Cutfield RG, Shienvold FL, Polonsky KS, Noel J, Olson L, Dillberger J, Miller J, Mintz DH (1986) Natural history of intrahepatic canine islet cell autografts. $\mathbf{J}$ Clin Invest 78 : 1339-1348

7. Child CG, Frey CJ, Fry WJ (1969) A reappraisal of removal of ninety-five per cent of the distal portion of the pancreas. Surg Gynecol Obstet 129:49-56

8. Sun AM, Coddling JA, Haist RE (1974) A study of glucose tolerance and insulin response in partially depancreatectomised dogs. Diabetes 23: 424-432

9. Franklin WA, Schulak JA, Reckard CR (1979) The fate of transplanted pancreatic islets in the rat. Am J Pathol 94:85-95

10. Sutherland DE (1981) Pancreas and islet transplantation. I. Experimental studies. Diabetologia 20:161-185

11. Scharp D, Lacy $\mathbf{P}(1985)$ Human islet isolation and transplantation. Diabetes [Suppl 1] 34: 5

12. Alejandro R, Mintz DH, Noel J, Latif Z, Koh N, Russell E, Miller J (1987) Islet cell transplantation in type 1 diabetes mellitus. Transplant Proc 19: 2359-2361

13. Tuch BE, Sheil AGR, Ng ABP, Turtle JR (1986) Transplantation of cultured human fetal pancreas into insulin-dependent diabetic humans. Transplant Proc 18: 260-263

14. Koulmanda M, Mandel TE (1987) Delayed reversal of diabetes after pancreas isotransplantation in chronically diabetic mice. Transplant Proc 19: 979-980

15. Cuthbertson RA, Koulmanda M, Mandel TE (1988) Detrimental effect of chronic diabetes on growth and function of fetal islet isografts in mice. Transplantation 46: 650-654

16. Mauer SM, Steffes MW, Sutherland DE, Najarian JS, Michael AF, Brown DM (1975) Studies of the rate of regression of the glomerular lesions in diabetic rats treated with pancreatic islet transplantation. Diabetes 24: 280-285

17. Chakrabarti S, Sima AA, Tze WJ, Tai J (1987) Prevention of diabetic retinal capillary pericyte degeneration and loss by pancreatic islet allograft. Curr Eye Res 6: 649-658

18. Gray DWR, Sutton R, McShane P, Peters M, Morris PJ (1986) Exocrine contamination adversely affects the implantation of rat islets transplanted to the kidney capsule site. Transplant Proc 18: 1823-1824

19. Tuch BE, Grigoriou S, Turtle JR (1988) Long-term passage of human fetal pancreas in non-diabetic nude mice fails to allow maturation of the response to glucose. Transplant Proc 20: 64-67

20. Imamura T, Koffler $M$, Helderman JH, Prince D, Thirlby $R$, Inman L, Unger RH (1988) Severe diabetes induced in subtotally depancreatectomized dogs by sustained hyperglycaemia. Diabetes 37: 600-609

Received: 14 February 1989

and in final revised form: 26 June 1989

Dr. D.W.R. Gray

Nuffield Department of Surgery

University of Oxford

John Radcliffe Hospital

Oxford OX3 9DU

UK 